\title{
Planning of Large Odontogenic Cysts Treatment Using Panoramic and CBCT Images
}

\author{
Saim Yanık ${ }^{1}$, Mehmet Emrah Polat ${ }^{1}$ \\ ${ }^{1}$ Department of Oral and Maxillofacial Surgery, Faculty of Dentistry, Harran University, Şanlıurfa, Turkey \\ Received: 21 November 2019, Accepted: 07 April 2020, Published online: 30 April 2020 \\ (C) Ordu University Institute of Health Sciences, Turkey, 2020
}

\begin{abstract}
Objective: We explored the utility of Cone Beam Computed Tomography (CBCT) and Orthopantomography $(\mathrm{OPG})$ in terms of treatment planning to determine which form of radiography can more reliably assess cyst volume.
\end{abstract}

Methods: 'We evaluated the panoramic and CBCT images of nine patients who consulted our clinic for treatment of large cystic lesions. Overall, 27 images were reviewed and analyzed by 21 oral and maxillofacial surgeons. We asked five questions (detailed in the main text).

Results: We evaluated the 189 answers in the questionnaire. The surgeons recommended marsupialization followed by enucleation, marsupialization, and enucleation (70.89\%, $14.28 \%$, and $14.81 \%$, respectively). The answers to the reasons of these treatment choices showed that the size of the cysts and relationship with the adjacent anatomical structures are the most effective factors. In after marsupialization challenges, $85.71 \%$ of the answers considered that the lesion had shrunk sufficiently to allow enucleation according to OPG's, however, this rate decreased to $42.23 \%$ when the same surgeons evaluated the CBCT images of the patients after marsupialization. Ninety-nine percent of the responses reported that CBCT was much more reliable than OPG.

Conclusion: In this study, we concluded that OPG imaging method can be used for the diagnosis and followup of cystic lesions, but in order to determine the accuracy of timing, adequacy of new bone formation and whether the cyst has shrunk sufficiently in volume, during the transition from the marsupialization process to the enucleation process, it is necessary to use CBCT imaging method. Further clinical trials should be conducted to define the effects of three dimensions images regarding surgical treatments of different kinds of oral and maxillofacial region cystic lesions.

Key words: $\mathrm{CBCT}$, panoramic, odontogenic cysts, marsupialization, enucleation

Suggested Citation: Yanik S, Polat ME. Planning of Large Odontogenic Cyst Treatment Using Panoramic and CBCT Images. Middle Black Sea Journal of Health Science, 2020; 6(1):45-53

Address for correspondence/reprints:

DOI: $\quad 10.19127 / \mathrm{mbsjohs.649495}$

Saim Yanık

Telephone number: +90 (414) 31830 00- 1968

ORCID-ID 0000-0002-1229-2982

E-mail: saimyanik@hotmail.com 


\section{Introduction}

Cysts are pathological cavities commonly found in the maxilla and mandible. Cysts feature a wall of connective tissue and are filled with epithelium or a liquid/semi-liquid. They are often localized in the jaws are often rich in epithelial tissue. Cysts are classified as odontogenic and non-odontogenic depending on the epithelium from which they originate (Rosai 2011). Cysts tend to expand and grow, causing resorption of bone tissue. Depending on the extent of resorption, cysts may cause severe damage such as bone fractures. There are many treatment options including enucleation, marsupialization, fenestration, and decompression. The treatment regimen is chosen upon the basis of cyst size, the extent of tissue damage, the availability of surgical access, patient's age, the proximity of the cyst to vital tissues, and the significance of the affected teeth in terms of eruption. In some cases, one approach is adequate; sometimes, several techniques are used in combination (Partsch 2005, Anavi et al., 2011, Wang et al., 2012). Marsupialization is the most minimally invasive procedure; a bony window is created in the cyst, facilitating new bone formation, reducing the cyst volume, and minimizing the iatrogenic neurovascular injury (Yahara et al., 2009). Cysts can be removed by enucleation alone, or enucleation may follow decompression or marsupialization, which reduces the cyst's size and the proximity of the cyst to important anatomical structures. Secondary surgery is less invasive than primary surgery; initial marsupialization or decompression followed by enucleation is widely recommended (Giuliani et al., 2006; Ecker et al., 2016).

Orthopantomography (OPG) is commonly used by oral and maxillofacial surgeons to evaluate vertical bone volume, to diagnose cysts and tumors, to evaluate maxillofacial trauma, and to identify dental caries and periodontal disease. OPG remains the gold standard in clinical practice. However, twodimensional radiographs can be affected by tissue superimposition attributable to a malocclusion deformity or other issues (Tang et al., 2017).

Cone-beam Computed Tomography (CBCT) is an improved form of Computed Tomography (CT) which introduced at the end of the 1990s to yield three-dimensional images of maxillofacial structures. CBCT is better than traditional CT in many aspects. CBCT reveals the relationships among impacted teeth, foreign bodies, supernumerary teeth, and anatomical structures; it also yields data on the mandibular condyle and peripheral bones. CBCT precedes dental implant surgery and other orthodontic procedures. CBCT optimally reveals root anomalies, dilatation, and "dens in dente" prior to endodontic treatment (Adibi et al., 2012).

The diagnosis of jawbone lesions is often difficult; such lesions are very variable. Imaging plays crucial roles in terms of detection, treatment, and follow-up. Although initial OPG remains the gold standard, CBCT is commonly used to create three-dimensional images. CBCT requires a higher radiation dose than OPG, but a lower dose than traditional CT (Shahidi et al., 2015). Thus, the purpose of this study was to evaluate the influence of $2 \mathrm{D}$ and $3 \mathrm{D}$ imaging techniques on the decision-making process of enucleation after marsupialization in large odontogenic cyst cases by questionnaire sent to 21 experienced oral and maxillofacial surgeons.

\section{Methods}

The Harran University Ethics Committee approved this study. (Date: 11/03/2019, Decision Number: HR/19.03.26.) We evaluated the OPG and CBCT images of nine patients admitted to a University Hospital in Turkey for the treatment of large cyst lesions. One female and eight male patients' radiographic and demographic records were included in this study and the mean age of patients was 26.33 years. The inclusion criteria were following; lesions of the maxilla or mandible at least $3 \mathrm{~cm}$ in diameter, preoperative OPG radiographs that indicated a high risk of iatrogenic damage during enucleation, the absence of any systemic disease such as a psychiatric conditions, diabetes mellitus, pregnancy, cardiovascular disease, or immunosuppression; and only patients with histopathological diagnosis of odontogenic cyst (Odontogenic Keratocyst n:3, Dentigerous Cyst n:3, Residual Cyst n:1, Radicular Cyst n:2 ). Also, patients who were imaged with CBCT simultaneously with the control OPG (film at the end of the marsupialization). There were 9 patients meeting these inclusion criteria.

Marsupialization treatment was initiated, and the patients were followed-up at two-week intervals. Patients were imaged with OPG approximately 3 months after the treatment to check the changes in the size of the cyst. When the lesion was evaluated as sufficiently shrunk, an appointment for enucleation was given to patient. In the first few patients, the cyst was found to be larger in size during the surgical procedure than it was estimated in OPG. It was observed that the lesion did not shrink sufficiently for enucleation. Then simultaneous CBCT was performed in subsequent patients when the cyst was evaluated as shrunken enough for enucleation 
according to the control OPG. The size of the cyst was compared in both films. And as a result of this comparison, it was observed that the cyst volume was greater than predicted in the control OPG film, both in CBCT records and clinically during enucleation. Then we decided to implement this study to examine if there is a difference between two imaging methods in terms of enucleation decision after marsupialization process.

\section{Study Design and Questionnaire}

We made a questionnaire survey, which includes questions regarding timing of treatment, reliability of two imaging methods and treatment choices (including reasons) of each patient with radiographic data, to 21 oral and maxillofacial surgeons who have at least 5 years of experience with CBCT and OPG diagnoses. The detailed version of the questionnaire is presented in Table 1. Totally, 27 images and 45 questions were reviewed by 21 oral and maxillofacial surgeons The surgeons evaluated 18 OPG images (9 OPG preoperative (Figure 1), 9 OPG at the end of marsupialization (Figure 2) and 9 CBCT (taken simultaneously with OPG's at the end of marsupialization (Figure 3). The surgeons also had knowledge of the odontogenic cyst pathologically diagnosed for all cases. The prepared electronic questionnaire was sent to 21 oral and maxillofacial surgeons by e-mail. This e-survey included 5 questions and 3 radiographic images for each patient. The answers of the survey statistically evaluated.

\section{Statistical Analyses}

The data were analyzed with IBM SPSS V23 (Chicago, USA). Analysis results were expressed as frequency and percentage for all categorical data. Chi-square test was used to compare categorical data. The significance level was taken as $\mathrm{p}<0.05$.

\section{Results}

The study includes nine patients (eight males and one female) with a mean age of 26.33 years (range, 13-49 years). All cysts underwent marsupialization followed by enucleation. The mean marsupialization time of the patients was calculated as $10.22( \pm 5.44)$ months. The 189 responses for the first question showed that the surgeons favored marsupialization followed by enucleation, marsupialization, and enucleation $(70.89,14.28$, and $14.81 \%$, respectively; Table 2). Regarding the factors affecting the treatment of choice, option a received 157 votes, option b 156, option c 104, option d 104, option e 85, option f 43, and option g 56. Of 189 answers (Table
2) to the third question, 162 were 'yes' and 27 were 'no'. Thus, $85.71 \%$ considered that the lesion had shrunk adequately to allow enucleation, and $14.29 \%$ did not (Table 2). For the fourth question (evaluation of the CBCT images), $57.67 \%$ (of 109 answers) were 'no'; the lesion had not sufficiently shrunk (Table 2). The proportion of 'yes' answers was significantly lower than that for question 3 (Table 3, p<0,05) For the last question, CBCT received 188 votes and OPG only 1; CBCT was more reliable than OPG (Table 2).

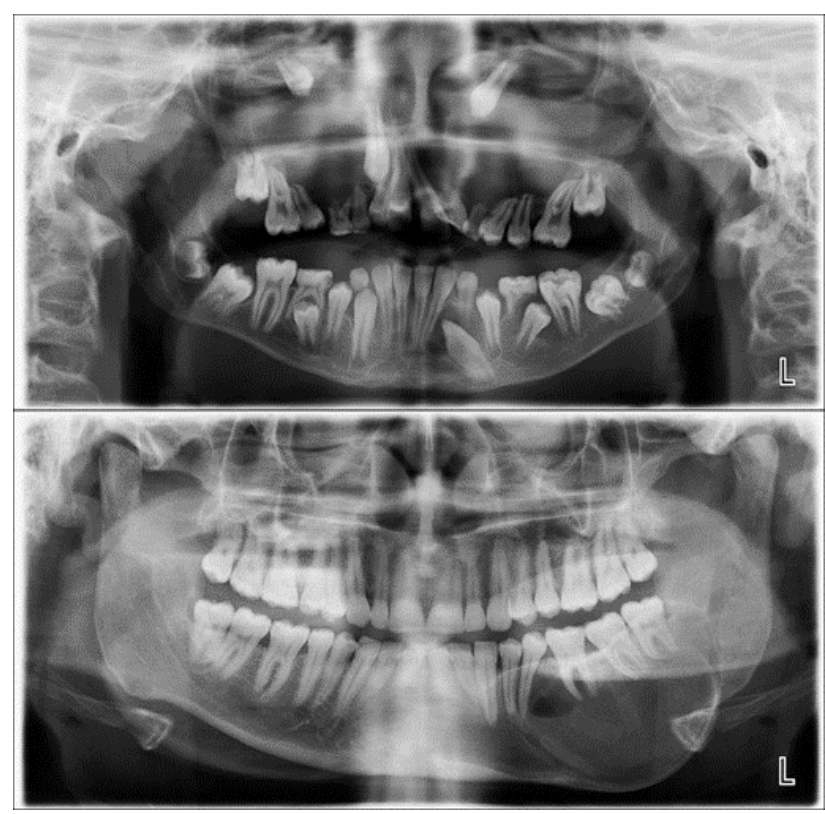

Figure 1. First OPG (Patients 1 and 2)
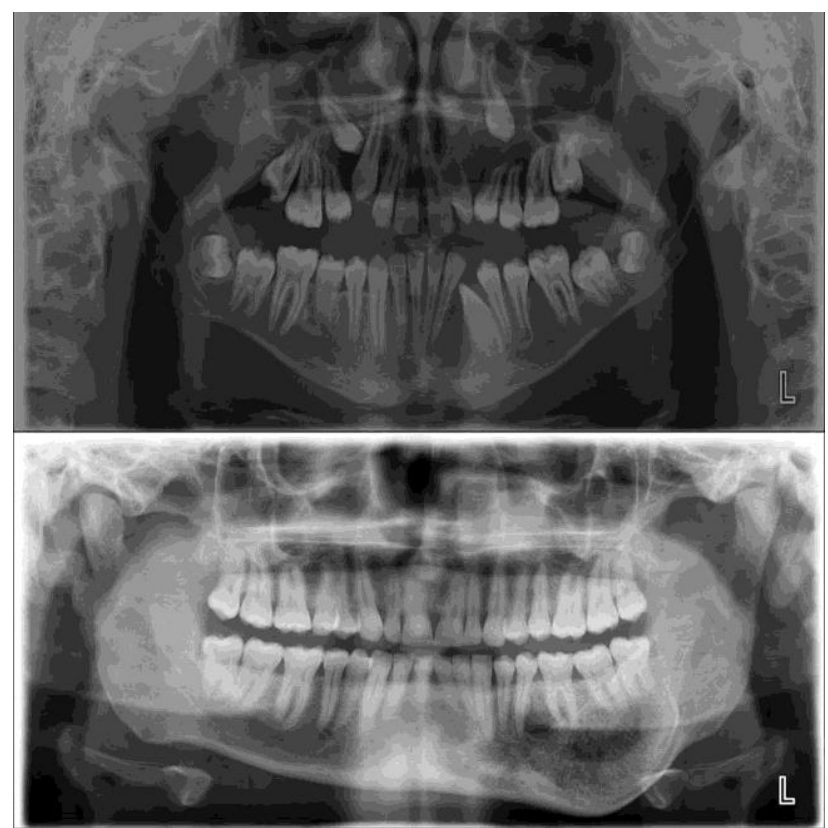

Figure 2. Control OPG taken after marsupialization treatment. (Patients 1 and 2). 


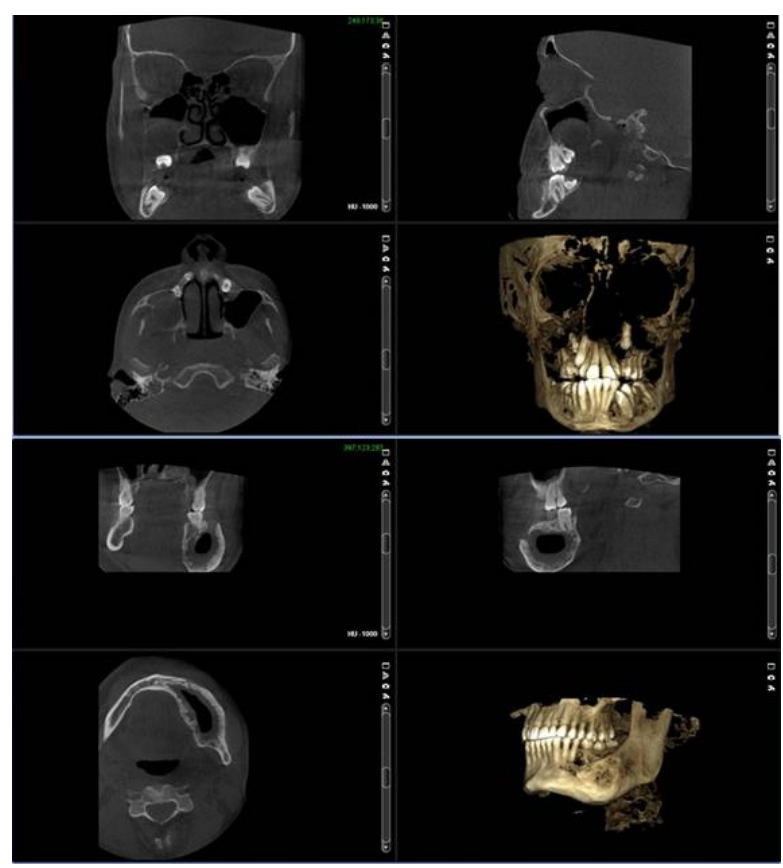

Figure 3. СBCT which was taken at the same time control panoramic film. (Patients 1 and 2)

Table 1. Questions and Options

\begin{tabular}{cc}
\hline Questions & Options \\
\hline $\begin{array}{c}\text { Q1 What is the best treatment option regarding the } \\
\text { first panoramic film? }\end{array}$ & $\begin{array}{c}\text { a)Marsualisation } \\
\text { b) Enucleation }\end{array}$ \\
\end{tabular}

a) The size of the cyst

b) The relationship between other anatomical features and the cyst, and the risk of damage to the regional anatomy

Q2 What prompted your answer to question 1?

c) The possibility of complete cyst removal via surgery

d) The possibility of fracture during or after surgery e) Patient age

f) The likelihood of maintaining teeth associated with the lesion

g) The likelihood that the tissue available for prosthetic treatment after enucleation will be inadequate

\begin{tabular}{|c|c|c|c|}
\hline Q3 & $\begin{array}{l}\text { Assume that you plan to treat the lesion via } \\
\text { marsupialization + enucleation. The lesion } \\
\text { must shrink maximally prior to enucleation, } \\
\text { by reference to the OPG film taken after } \\
\text { marsupialization. Is the cyst sufficiently } \\
\text { shrunken to allow enucleation? }\end{array}$ & 1) Yes & 2) $\mathrm{No}$ \\
\hline Q4 & $\begin{array}{l}\text { Looking at the CBCT scan taken at the same } \\
\text { time as the OPG film, has the cyst shrunk } \\
\text { sufficiently to allow enucleation? }\end{array}$ & 1) Yes & 2) $\mathrm{No}$ \\
\hline Q5 & $\begin{array}{l}\text { Which of the following imaging modalities } \\
\text { more reliably identify the boundaries of the } \\
\text { lesion and the optimal treatment? }\end{array}$ & 1) OPG film & 2) $\mathrm{CBCT}$ film \\
\hline
\end{tabular}


Table 2. Questions and vote distrubutions

\begin{tabular}{|c|c|c|c|}
\hline \multicolumn{2}{|c|}{ Questions } & $\mathrm{n}$ & $\%$ \\
\hline \multirow{3}{*}{ Q1 } & Marsupialization + enucleation & 134 & 70,9 \\
\hline & Marsupialization & 27 & 14,3 \\
\hline & Enucleation & 28 & 14,8 \\
\hline \multirow{7}{*}{ Q2 } & a & 157 & 83,1 \\
\hline & b & 156 & 82,5 \\
\hline & c & 104 & 55,0 \\
\hline & $d$ & 104 & 55,0 \\
\hline & e & 85 & 45,0 \\
\hline & $f$ & 43 & 22,8 \\
\hline & g & 56 & 29,6 \\
\hline \multirow[b]{2}{*}{ Q3 } & Yes & 162 & 85,7 \\
\hline & No & 27 & 14,3 \\
\hline \multirow{2}{*}{ Q4 } & Yes & 109 & 57,7 \\
\hline & No & 80 & 42,3 \\
\hline \multirow{2}{*}{ Q5 } & СBCT & 188 & 99,0 \\
\hline & Panaromic & 1 & 1,0 \\
\hline
\end{tabular}

Table 3. Statistical analyses of the 3th and 4th questinairres.

\begin{tabular}{|c|c|c|c|}
\hline Questinnaires & \multicolumn{2}{|c|}{ Panoramic } & $\mathbf{p}$ \\
\hline \multirow{3}{*}{$\begin{array}{l}\text { Q3. Assume that you plan to treat the lesion via } \\
\text { marsupialization + enucleation. The lesion must shrink maximally } \\
\text { prior to enucleation, by reference to the OPG film taken after } \\
\text { marsupialization. Is the cyst sufficiently shrunken to allow } \\
\text { enucleation? }\end{array}$} & Yes & No & \multirow{5}{*}{$<0.001$} \\
\hline & 162 & 27 & \\
\hline & \multicolumn{2}{|c|}{ CBCT } & \\
\hline \multirow{2}{*}{$\begin{array}{l}\text { Q4. Looking at the CBCT scan taken at the same time as the } \\
\text { OPG film, has the cyst shrunk sufficiently to allow enucleation? }\end{array}$} & Yes & No & \\
\hline & 80 & 109 & \\
\hline
\end{tabular}

$\mathrm{P}$ value of $<0.05$ was considered statistically significant

\section{Discussion}

In this retrospective study, we evaluated the optimal radiographic imaging modality in terms of surgical planning and evaluation of cyst volume after marsupialization.

In previous studies, the authors compared CBCT and OPG on many different aspects such as: surgical technique of maxillary and mandibular third molar, classifying of lower third molar, upper molar root protrusion into maxillary sinus, relation of impacted mandibular third molars to the inferior alveolar nerve, presurgical evaluation of bony implant sites, impacted maxillary canines, and anatomical characteristics and visibility of mental foramen and accessory mental foramen (Ghaeminia et al.,2009; Aravindaksha et al., 2015; Lorenzo et al., 2015; Pico et al., ;2017; Araujo et al., 2019; Hermann et al., 2019; Brasil et al., 2019; Themkumkwun et al., 2019; Nirmalendu et al., 2019; Fortes et al., 2019). None of the prior studies compared CBCT and OPG films taken after the marsupialization of odontogenic cysts in terms of evaluating whether the odontogenic cyst volume allows enucleation.

Although various treatments are used for the surgical removal of large odontogenic cysts, no standard protocol is available. Treatment seeks 
complete removal of the lesion and minimization of postoperative complications. Cystectomy, accounting for the entire enucleation of the lesion, is a technique which is faster than marsupialization, but it has some disadvantages such as damaging the important anatomical structures nearby the lesions (Lizio et al. 2013; Wakolbinger et al. 2016). Wakolbinger et al. and Lizio et al. offer marsupialization, followed by enucleation in such cases to diminish these risks. Marsupialization is a technique described by Partsch, 2005 , reduces the intra-cystic pressure and induces new bone formation. In this technique, the communication path between the oral cavity and the lesion is conducted with the use of various devices (tube, stent). In this study, serum drainage tube was used to maintain oral and intracystic connection. Marsupialization allows the fulfilment of both aims and is a useful conservative treatment for large jaw cysts (Lee et al., 2017). Marsupialization followed by enucleation minimizes anatomical damage in the patients with odontogenic cysts, associated with few postoperative complications and a better quality of life. The cyst volume reduces gradually; there is no risk of jaw fracture, adjacent tissues are protected and also it is suggested that decompression followed by enucleation has a lower relapse rate compared with enucleation alone (Shudou et al., 2012; Oliveros et al., 2017).

Although various treatments are used for the surgical removal of large odontogenic cysts, no standard protocol is available. Treatment seeks complete removal of the lesion and minimization of postoperative complications. Cystectomy, accounting for the entire enucleation of the lesion, is a technique which is faster than marsupialization, but it has some disadvantages such as damaging the important anatomical structures nearby the lesions (Lizio et al. 2013; Wakolbinger et al. 2016). Wakolbinger et al. and Lizio et al. offer marsupialization, followed by enucleation in such cases to diminish these risks. Marsupialization is a technique described by Partsch, 2005, reduces the intra-cystic pressure and induces new bone formation. In this technique, the communication path between the oral cavity and the lesion is conducted with the use of various devices (tube, stent). In this study, serum drainage tube was used to maintain oral and intracystic connection. Marsupialization allows the fulfilment of both aims and is a useful conservative treatment for large jaw cysts (Lee et al., 2017). Marsupialization followed by enucleation minimizes anatomical damage in the patients with odontogenic cysts, associated with few postoperative complications and a better quality of life. The cyst volume reduces gradually; there is no risk of jaw fracture, adjacent tissues are protected and also it is suggested that decompression followed by enucleation has a lower relapse rate compared with enucleation alone (Shudou et al., 2012; Oliveros et al., 2017).

The surgeons were asked to choose an optimal treatment, and then to explain the reason for their choice (from the several possible reasons listed in the question). Of the 189 replies, marsupialization followed by enucleation, marsupialization, and enucleation received $70.89,14.28$, and $14.81 \%$ of the votes, respectively (first question). In terms of the reasons behind the choice, option a received 157 votes, option b 156, option c 104, option d 104, option e 85, option f 43, and option g 56 (second question). The preferred treatment was marsupialization followed by enucleation; marsupialization alone and enucleation alone received similar (low) numbers of votes. For the reason of the two-step treatment, cyst size and possible damage to nearby anatomical structures were of concern to the majority of surgeons, who also thought that complete cyst removal might be difficult without marsupialization, thus associated with a fracture risk. The surgeons were not concerned about the patient's age, tooth condition, or possible difficulties with prosthetic treatment after enucleation. In general, the authors agreed that if a cystic lesion was large, laying adjacent to an important anatomical structure, involving a tooth, or was associated with a fracture risk, marsupialization followed by enucleation should be preferred.

This approach (marsupialization followed by enucleation) has certain disadvantages; patient compliance is essential in terms of careful oral hygiene, daily irrigation of the drain and the surrounding tissues, and several follow-up visits. The tube entrance may become blocked, the tube may be lost, and further treatment may be required prior to enucleation (Oliveros et al., 2017). Indeed, lost tubes, patients who do not keep follow-up appointments and irrigation problems were encountered in this study. All patients underwent enucleation after marsupialization.

Radiography plays a significant role in the diagnosis of odontogenic cysts; they are commonly found incidentally on routine radiographic examinations. Conventional radiographs (periapical and panoramic films) are useful and are routinely taken; CT and CBCT radiographs are essential for correct diagnosis and treatment by oral and maxillofacial surgeons (Ghaeminia et al., 2009). Plain radiographs are sometimes unreliable because of the superimposition of anatomical structures, ghost 
images, and/or horizontal and vertical distortions (Bouquet et al., 2004). CBCT requires a little more radiation than does OPG but far less than CT; further, CBCT is useful and practicable (Ludlow et al., 2008). CBCT delivers full-size three-dimensional images; axial, coronal, and sagittal sections may be viewed separately if required. CBCT is of great assistance preoperatively, for surgical planning, and during the follow-up of jawbone lesions (Bouquet et al., 2004; Prabhusankar 2014).

The studies regarding surgical planning and imaging methods were present in the literature about tooth removal. Most of these studies stated that there were no statistically significant differences in surgical planning when panoramic and CBCT images were compared before surgery (Aravindaksha et al., 2015). Aravindaksha et al. (2015) assessed the impact of CBCT images on mandibular third molar removal in terms of elevator placement, tooth sectioning, direction of tooth removal and removal of buccal bone. They stated that the CBCT images did not change the surgical treatment plan in mandibular third molar removal. In addition, Araujo et al. (2019) reported that no statistically significant changes were present between CBCT and panoramic images in mandibular third molar removal. Nevertheless, Ghaeminia et al. (2009) reported that CBCT images before mandibular third molar surgery had a dramatic effect in surgical planning in the direction of tooth removal regarding buccolingual relationship between tooth and mandibular canal. Also, Hermann et al (2019) determined that CBCT images changed the treatment plan regarding external root resorption compared to panoramic images. According to Hermann el al. (2019) decision to remove second molars are more often encountered after CBCT examinations. Brasil et al. (2019) performed a comparison of panoramic and CBCT findings for classification of impacted lower third molars according to the parameters of the Pell and Gregory classification. In their study, two oral and maxillofacial radiologists evaluated $\mathrm{OPG}$ and $\mathrm{CBCT}$ images and as a result of this study OPG shows similar performance compared with CBCT in the classification of impaction. Themkumkwun et al. (2019) conducted a study to determine correlation between Panoramic and CBCT images in terms of upper molar root protrusion into the maxillary sinus and they highlighted that there was a respective correlation between two imaging methods. In a study evaluating the relationship between IAN and impacted lower molar roots in terms of OPG and CBCT imaging methods, it was reported that CBCT was more effective in evaluating the relationship of these anatomical structures (Nirmalendu et al., 2019). The study conducted by Fortes et al. (2019) assessed the influence of 2D and 3D imaging methods on implant surgery planning. According to their results, the linear measurements in Panoramic radiography was not accurate and image magnification-distortion were expected and vertical measurements in Panoramic radiography might result with overestimation. Lorenzo et al. (2015) aimed to compare the skills of CBCT and OPG in terms of mental foramen and accessory mental foramen visualization. According to their results $83.87 \%$ of the mental foramens and $45.83 \%$ of the accessory mental foramens identified on CBCT were also visible on panoramic images. A questionnaire implemented by Pico et al. (2017) reported that the CBCT imaging gave further information when compared to panoramic radiography regarding the impacted upper canine assessment. This questionnaire was performed with nine orthodontists and they were asked to analyze different topics regarding the impacted maxillary canines.

According to these studies, the superiority of CBCT over OPG is still controversial. While most of the authors stated that CBCT was superior, some stated that there was no difference between the two imaging techniques. In this study, the experienced surgeons were asked "Is the cyst sufficiently shrunken (on the OPG film) to allow enucleation?". Overall, $85.71 \%$ of the respondents replied 'yes'. We then asked the same question but referred the surgeons to the CBCT film; $57.67 \%$ replied 'no'. This result means that; $85.71 \%$ of surgeons stated that cysts had shrunk enough for enucleation according to OPG, whereas this rate decreased to $42.33 \%$ when the same surgeons evaluated the same patients' CBCT images taken simultaneously with the control OPG. Thus, CBCT revealed the real cyst size by accurately defining the boundaries thereof; a majority of surgeons felt that more time should pass prior to enucleation. Almost all surgeons stated that CBCT was more reliable than OPG.

One of the limitations of this study was defining the surgeons with 5 years of professional background as experienced. In fact, experience should be based not only on the number of years studied but on more subjective data such as the number of operations performed or complication rates. In previous studies the examiners' experienced term was explained in detail but Lambert et al., (1997) defined professionals' experience level by the number of implants they had placed previously in an implant planning study. 
Although the effects of CBCT and panoramic imaging systems on surgical techniques have been investigated in the literature, no studies on the surgery of odontogenic cysts have been found. In the present study, the surgical planning of large odontogenic cysts is clearly changed when experienced surgeons' answers were evaluated.

\section{Conclusion}

In conclusion, it seems that examination of the cyst volume by CBCT may change the final treatment decision at the end of the marsupialization process. CBCT and OPG data differed significantly when the techniques were used to define size of cystic lesions. In this study, we concluded that OPG imaging method can be used for the diagnosis and follow-up of cystic lesions, but in order to determine the accuracy of timing, adequacy of new bone formation and whether the cyst has shrunk sufficiently in volume, during the transition from the marsupialization process to the enucleation process, it is necessary to use CBCT imaging method. Further clinical trials should be conducted to define the effects of three dimensions images regarding surgical treatments of different kinds of oral and maxillofacial region cystic lesions.

Ethics Committee Approval: Ethics committee approval was received for this study from Nonclinical Research Ethics Committee of Harran University Medical Faculty. (Date: 11/03/2019, Decision Number: HR/19.03.26.)

Peer-review: Externally peer-reviewed.

Author Contributions: Concept- S.Y., M.E.P, Design M.E.P., S.Y; Supervision- M.E.P.; Materials M.E.P.; Data Collection/Data Process- M.E.P.; Analyze or Comment-S.Y; Literature Scanning-S.Y; Writer of Paper-S.Y., M.E.P; Critical ReviewM.E.P., S.Y.

Conflict of Interest: No conflict of interest was declared by the authors.

Financial Disclosure: The authors declared that this study hasn't received no financial support.

\section{References}

Adibi S, Zhang W, Servos T, O'Neill PN.Cone beam computed tomography indentistry: what dental educators and learners should know. J Dent Educ 2012;76:1437-42.

Anavi Y, Gal G, Miron H, Calderon S, Allon DM. Decompression of odontogenic cystic lesions: clinical long-term study of 73 cases. Oral Surg Oral Med Oral Pathol Oral Radiol Endod 2011;112:164-9.
Araujo GTT, Peralta-Mamani M, Silva AFMD, Rubira CMF, Honório HM, Rubira-Bullen IRF. Influence of cone beam computed tomography versus panoramic radiography on the surgical technique of third molar removal: a systematic review. Int. J. Oral Maxillofac. Surg 2019;48:1340-7.

Aravindaksha SP, Balasundaram A, Gauthier B, Pervolarakis T, Boss H, Dhawan A, et al. Does the use of cone beam CT for the removal of wisdom teeth change the surgical approach compared with panoramic radiography? J Oral Maxillofac Surg 2015;73:e12.

Bouquet A, Coudert JL, Bourgeois D, Mazoyer JF, Bossard D. Contributions of reformatted computed tomography and panoramic radiography in the localization of third molars relative to the maxillary sinus. Oral Surg Oral Med Oral Pathol Oral Radiol Endod 2004;98.342 347.

Brasil DM, Nascimento EHL, Gaêta-Araujo H, Oliveira-Santos C, Maria de Almeida S. Is Panoramic Imaging Equivalent to Cone-Beam Computed Tomography for Classifying Impacted Lower Third Molars?. J Oral Maxillofac Surg 2019;1-7.

Ecker J, Horst RT, Koslovsky D. Current role of Carnoy's solution in treating keratocystic odontogenic tumors. J Oral Maxillofac Surg 2016;74:278-82.

Fortes JH, Santos CO, Matsumoto W, Motta RJG, Tirapelli $C$. Influence of $2 \mathrm{D}$ vs $3 \mathrm{D}$ imaging and professional experience on dental implant treatment planning. Clinical Oral Investigations 2019; 23:929-36.

Ghaeminia H, Meijer GJ, Soehardi A, Borstlap WA, Mulder J, Bergé SJ. Position of the impacted third molar in relation to the mandibular canal, Diagnostic accuracy of cone beam computed tomography compared with panoramic radiography. Int. J. Oral Maxillofac Surg 2009; 38: 964-71.

Giuliani M, Grossi GB, Lajolo C. Conservative management of a large odonto- genic keratocyst: report of a case and review of the literature. J Oral Maxillofac Surg 2006;64:308-16.

Hermann L, Wenzel A, Schropp L, Matzen LH. Impact of cbct on treatment decision related to surgical removal of impacted maxillary third molars: does cbct change the surgical approach? Dentomaxillofacial Radiology 2019;48: 20190209. 
Lambert P, Morris H, Ochi S. Positive effect of surgical experience with implants on second stage implant survival. JOralMaxillofac Surg 1997;55:12-8.

Lee ST, Kim SG, Moon SY, Oh JS, JS You, Kim JS. The effect of decompression as treatment of the cysts in the jaws: retrospective analysis. J Korean Assoc Oral Maxillofac Surg 2017;43:83-7.

Lizio G, Sterrantino AF, Ragazzini S, Marchetti C. Volume reduction of cystic lesions after surgical decompression: A computerised threedimensional computed tomographic evaluation. Clin Oral Investig 2013;17:1701-8.

Lorenzo JM, Quintanilla JAS, Alonso AF, Mallou JV, Cunqueiro MMS. Anatomical characteristics and visibility of mental foramen and accessory mental foramen: Panoramic radiography vs. cone beam CT. Med Oral Patol Oral Cir Bucal 2015:1;20 (6):707-14.

Ludlow JB, Ivanovic M. Comparative dosimetry of dental CBCT devices and 64-slice CT for oral and maxillofacial radiology, Oral Surg Oral Med Oral Pathol Oral Radiol Endod 2008;106:1.106-14.

Nirmalendu S, Kedarnath NS, Singh $M$. Orthopantomography and Cone-Beam Computed Tomography for the Relation of Inferior Alveolar Nerve to the Impacted Mandibular Third Molars. Annals of Maxillofacial Surgery 2019;9(1):4-9.

Oliveros-Lopez L, Fernandez-Olavarria A, TorresLagares D, Serrera-Figallo MA, Castillo-Oyagüe $\mathrm{R}$, Segura-Egea JJ, et al. Reduction rate by decompression as a treatment of odontogenic cysts. Med Oral Patol Oral Cir Bucal 2017;22:643-50.

Partsch C. Treatment of keratocysts: The case for decompression and marsupialization. $\mathbf{J}$ Oral Maxillofac Surg 2005;63:1667-73.

Pico CL, do Vale FJ, Caramelo FJ, Corte-Real A, Pereira SM. Comparative analysis of impacted upper canines: Panoramic radiograph vs Cone Beam Computed Tomography. J Clin Exp Dent. 2017; 9: e1176-82.

Prabhusankar K, Yuvaraj A, Prakash CA, Parthiban J, Praveen B. CBCT Cyst Leasions Diagnosis Imaging Mandible Maxilla. J Clin Diagn Res 2014;8:3-5.

Rosai J. Rosai and Ackerman's Surgical Pathology. 2011;265-7.

Shahidi S, Shakibafard A, Zamiri B, Mokhtare MR, Houshyar M, Mahdianf S. The feasibility of ultrasonography in defining the size of jaw osseous lesions. J Dent (Shiraz) 2015;16:335-40.
Shudou H, Sasaki M, Yamashiro T, Tsunomachi S, Takenoshita Y, Kubota Y, et al. Marsupialisation for keratocystic odontogenic tumours in the $\mathrm{m}$ andible: longitudinal image analysis of tumour size using 3D visualised CT scans. Int. J Oral Maxillofac Surg 2012;41:290-6.

Tang Z, Liu X, Chen K. Comparison of digital panoramic radiography versus cone beam computerized tomography for measuring alveolar bone. Head \& Face Medicine 2017; 13:2

Themkumkwun S, Kitisubkanchana J, Waikakul A, Boonsiriseth K. Maxillary molar root protrusion into the maxillary sinus: a comparison of cone beam computed tomography and panoramic findings. Int. J. Oral Maxillofac. Surg 2019;48:1570-6

Wakolbinger R, Beck-Mannagetta J. Long-term results after treatment of extensive odontogenic cysts of the jaws: a review. Clin Oral Investig 2016;20:15-22.

Wang Y, He D, Yang C, Wang B, Qian W. An easy way to apply orthodontic extraction for impacted lower third molar compressing to the inferior alveolar nerve. J Craniomaxillofac Surg 2012;40:234-7.

Yahara Y, Kubota Y, Yamashiro T, Shirasuna K.Eruption prediction of mandibular premolars associated with dentigerous cysts. Oral Surg Oral Med Oral Pathol Oral Radiol Endod 2009;108:2831. 